\title{
Physician Assessment of Pretest Probability of ( Malignancy and Adherence With Guidelines for Pulmonary Nodule Evaluation
}

Nichole T. Tanner, MD, MSCR, FCCP; Alexander Porter, MD; Michael K. Gould, MD, FCCP; Xiao-Jun Li, PhD; Anil Vachani, MD, FCCP; and Gerard A. Silvestri, MD, FCCP

BACKGROUND: The annual incidence of pulmonary nodules is estimated at 1.57 million. Guidelines recommend using an initial assessment of nodule probability of malignancy (pCA). A previous study found that despite this recommendation, physicians did not follow guidelines.

METHODS: Physician assessments $(\mathrm{N}=337)$ and two previously validated risk model assessments of pretest probability of cancer were evaluated for performance in 337 patients with pulmonary nodules based on final diagnosis and compared. Physician-assessed pCA was categorized into low, intermediate, and high risk, and the next test ordered was evaluated.

RESULTS: The prevalence of malignancy was $47 \%(n=158)$ at 1 year. Physician-assessed pCA performed better than nodule prediction calculators (area under the curve, 0.85 vs 0.75 ; $P<.001$ and .78; $P=.0001$ ). Physicians did not follow indicated guidelines when selecting the next test in $61 \%$ of cases $(n=205)$. Despite recommendations for serial CT imaging in those with low pCA, $52 \%(\mathrm{n}=13)$ were managed more aggressively with PET imaging or biopsy; $12 \%(\mathrm{n}=3)$ underwent biopsy procedures for benign disease. Alternatively, in the high-risk category, the majority $(\mathrm{n}=103[75 \%])$ were managed more conservatively. Stratified by diagnosis, $92 \%(\mathrm{n}=22)$ with benign disease underwent more conservative management with CT imaging (20\%), PET scanning (15\%), or biopsy (8\%), although three had surgery (8\%).

CONCLUSIONS: Physician assessment as a means for predicting malignancy in pulmonary nodules is more accurate than previously validated nodule prediction calculators. Despite the accuracy of clinical intuition, physicians did not follow guideline-based recommendations when selecting the next diagnostic test. To provide optimal patient care, focus in the areas of guideline refinement, implementation, and dissemination is needed.

CHEST 2017; 152(2):263-270

KEY WORDS: guideline adherence; nodule management; nodule risk prediction; pulmonary nodules

ABBREVIATIONS: $\mathrm{AUC}=$ area under the curve; $\mathrm{pCA}=$ probability of malignancy

AfFiliations: From the Thoracic Oncology Research Group (Drs Tanner and Silvestri), Division of Pulmonary and Critical Care, Medical University of South Carolina, and the Health Equity and Rural Outreach Innovation Center (Dr Tanner), Ralph H. Johnson Veterans Affairs Hospital, Charleston, SC; Integrated Diagnostics, Inc. (Drs Porter and Li), Seattle, WA; Kaiser Permanente Southern California (Dr Gould), Pasadena, CA; Pulmonary, Allergy, and Critical Care
Division (Dr Vachani), Department of Medicine, Perelman School of Medicine, University of Pennsylvania, Philadelphia, PA.

FUNDING/SUPPORT: This work was supported by Integrated Diagnostics, Inc.

CORRESPONDENCE TO: Nichole T. Tanner, MD, MSCR, 96 Johnathan Lucas St, CSB 816, Charleston, SC 29412; e-mail: tripici@musc.edu Published by Elsevier Inc. under license from the American College of Chest Physicians.

DOI: http://dx.doi.org/10.1016/j.chest.2017.01.018 
The estimated annual incidence of pulmonary nodules detected on chest CT imaging in the United States is 1.6 million cases. ${ }^{1}$ This is in large part due to scans being increasingly ordered for a variety of clinical presentations. In one large integrated health system, the number of scans performed over a 7-year period increased by $53 \% .{ }^{1}$ This calculation, which was done before the advent of lung cancer screening, is likely to increase further as screening is implemented in the United States. ${ }^{1}$

The management of pulmonary nodules is driven by the importance of distinguishing those that are malignant from those that are not by balancing the desire to intervene quickly for malignant nodules while avoiding and limiting procedures for ones that are benign. Guidelines for pulmonary nodule management encourage physicians to use estimates of pretest probability for malignancy as determined by clinical intuition or validated prediction models. ${ }^{2,3}$ Based on this risk assessment, guidelines recommend surveillance in nodules with low risk, PET imaging \pm biopsy in those with intermediate risk, and surgery in those at high risk.

Despite guideline recommendations, however, a previous study of nodules managed by community pulmonologists found that biopsy and surgery rates were no different between patients at low, intermediate, and high risks of malignancy. ${ }^{4}$ This study was limited by the inability to obtain clinician-assessed pretest probability at the time of the management decision. Therefore, no direct link between physician-documented knowledge of pretest probability of cancer and downstream decisionmaking could be established. We undertook this study to prospectively compare intuitive estimates of pretest probability to validated nodule malignancy prediction calculators. In addition, we sought to determine how often appropriate guideline-recommended diagnostic testing was used based on clinician assessment of malignancy.

\section{Methods}

These data were collected as part of a prospective multicenter observational trial evaluating a plasma proteomics test for pulmonary nodules. It was approved by the Institutional Review Board of the Medical University of South Carolina (Pro00020287).

\section{Site Selection}

Thirty-three North American (31 US, two Canadian) sites qualified and agreed to participate. Three hundred thirty-seven physicians participated in the trial: 278 pulmonologists, 47 surgeons, and 12 oncologists.

\section{Patient Selection}

Eligibility included patients $\geq 40$ years of age referred to a pulmonologist or a thoracic surgeon, or both, for evaluation of a lung nodule $\geq 8 \mathrm{~mm}$ and $\leq 30 \mathrm{~mm}$ identified on a baseline CT scan and first detected within 60 days of enrollment. Those with CT scans identifying the same nodule $>60$ days prior to enrollment and those with a current or prior diagnosis of any cancer within the previous 2 years, with the exception of nonmelanomatous skin cancer, were excluded. Consecutive patients meeting criteria and agreeing to participate were enrolled.

\section{Data Collection}

Patient data, including demographics, imaging, and procedures performed, were collected until a definitive diagnosis, nodule resolution, or at least 1 year of radiographic stability. At the time of enrollment, the treating physician was asked to indicate his/her assessment of nodule pretest probability of malignancy (pCA) (eg, 0\%-5\%, 6\%-10\%, 11\%-20\%, 21\%-30\%). Physician assessment of malignancy was individually determined, taking into account patient and nodule characteristics. Nodule characteristics, including size, contour, and location as determined by the interpreting radiologist, were documented on baseline and subsequent scans. Data were collected in patients who underwent subsequent evaluation with PET scanning. These scans were further categorized as those performed within 30 days of surgery and those not associated with surgery. Data on procedure use, including bronchoscopy, transthoracic needle biopsy, and surgery, were collected.

\section{Pretest $p C A$}

Two previously developed and validated models were used to estimate the pCA in each patient. ${ }^{5,6}$ Model accuracy and clinician-estimated pretest probabilities were determined by comparing the pCA with the final diagnosis. Receiver operating characteristic curves and the area under the curve (AUC) were generated for both models and physician pCA with 95\% CIs. Differences between the AUCs were calculated using standard error incorporating covariance and large sample Wald approximation. Physician-provided pCA for each patient was categorized into three groups based on the 2007 American College of Chest Physician (ACCP) guidelines for pulmonary nodule management ( $<5 \%, 5 \%-60 \%$ and $>60 \%)$. Procedure use by categories of risk was examined for the next test ordered after the baseline CT scan identifying the nodule and the most invasive test ordered over the entire course of nodule management.

\section{Data Analysis}

$\chi^{2}$ or analysis of variance tests were used to compare subgroups, and $P$ values of .05 were considered significant. A nodule was classified as benign based on confirmed benign pathologic features, radiographic resolution, or the absence of growth seen on radiographs as determined by the managing physician during surveillance of at least 1 year and up to 2 years. Statistical analyses were performed using MATLAB, version 8.3.0.532 (MathWorks) and MedCalc, version 16.4 (MedCalc Software). 


\section{Results}

A total of 685 patients were enrolled in this multicenter prospective observational study. Figure 1 shows the reasons patients were excluded from the final analysis. Results are reported for the final cohort of 337 patients.

The prevalence of cancer in this cohort was $47 \%(n=158)$. The demographics of participants stratified by diagnosis are presented in Table 1 . The average age of all participants was 66 years ( \pm 1.1 year) with a relatively even distribution of men and women. The majority (82\%) were ever smokers with an average pack-year smoking history of 45.4 ( \pm 3.8$)$. Fifty-four (16\%) had a previous history of cancer. There was no difference in the average pack-years between subjects with malignant nodules and those with benign nodules, although those with benign causes were twice as likely to be never smokers as opposed to those with malignancy. Twenty-one percent of nodules $\leq 11 \mathrm{~mm}$ in size were malignant, whereas $68 \%$ of nodules between 15 and $30 \mathrm{~mm}$ were malignant $(P<.001)$ (e-Fig 1). The majority of diagnosed malignant nodules were primary lung cancers, with only one metastatic melanoma.

To better compare clinician baseline pCA assessment to prediction models, we calculated pretest probability for each individual in the study using two previously validated models (e-Appendix 1). Physician assessment of pCA was concordant with Veterans Administration (VA)- and Mayo-model-calculated pCA in $64 \%$ and $65 \%$ of cases, respectively (e-Table 1 ).

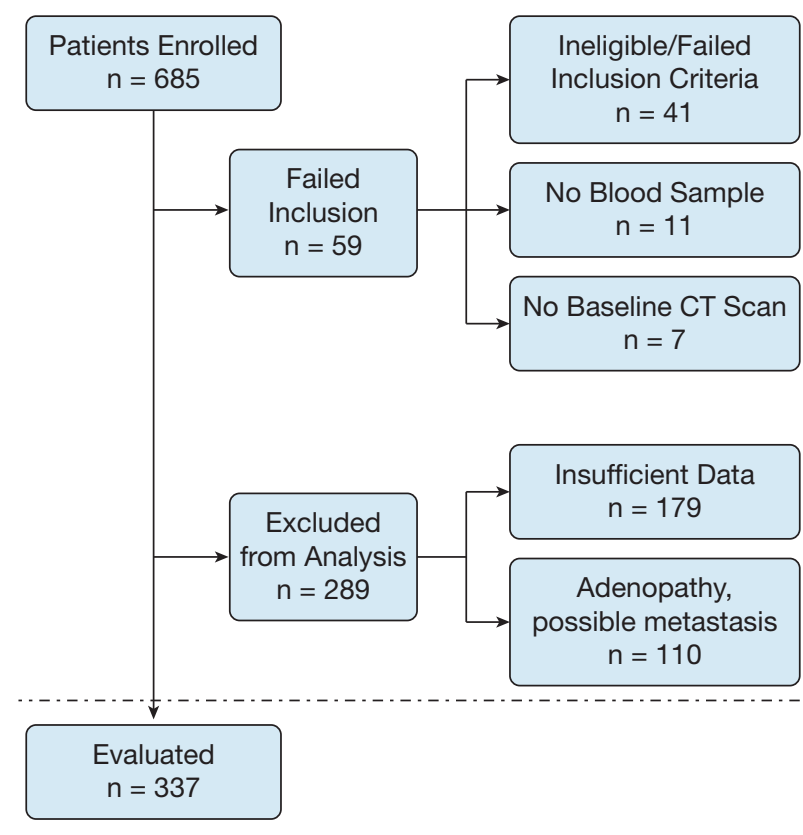

Figure 1 - Consort diagram.
Physicians were significantly better at predicting malignancy than either model (AUC, 0.85 vs 0.75 [VA model]; 95\% CI, 0.70- 0.79 and AUC, 0.78 [Mayo model], 95\% CI, 0.73- 0.82) (Fig 2). Physician-indicated pretest probability (pCA) at baseline CT scanning was categorized based on the 2007 version of the American College of Chest Physicians guidelines for nodule management, including low $\mathrm{pCA}<5 \%$, intermediate pCA $5 \%$ to $60 \%$, and high pCA $>60 \%$. Despite more accurately predicting malignancy, physician management differed from guideline recommendations on the next step in nodule evaluation in $61 \%$ of cases $(n=205)$. More than half of nodules ( $\mathrm{n}=177$ [53\%]) were managed more conservatively, whereas $8 \%(n=28)$ were managed more aggressively compared with guideline recommendations.

Of the 25 nodules in which physician pCA was $<5 \%$, the vast majority $(n=22)$ were ultimately found to be benign (Table 2). In this risk category, for which CT surveillance is recommended, management strategies were more aggressive in $52 \%$ of cases $(n=13)$, with three patients (12\%) undergoing biopsy for benign disease. The three patients diagnosed with malignancy underwent PET scanning as the first test. Physician-assessed pCA placed 138 nodules in the high-risk $(>0.60)$ category; $114(83 \%)$ were ultimately diagnosed as malignant. Although guidelines recommend surgery as the next step for most patients in this category, the majority $(\mathrm{n}=103$ [75\%]) were managed more conservatively (Table 2 ). Among those with benign disease, $92 \%(\mathrm{n}=22)$ underwent more conservative management with serial CT scanning (20\%), PET scanning (63\%), or biopsy (8\%), and ultimately only two patients (8\%) went on to have surgery for benign disease. Of those with malignancy, 33 (29\%) went straight to guideline-recommended surgery, whereas 81 (71\%) were managed by serial CT imaging (4\%), PET scanning (55\%), or biopsy (11\%). VA- and Mayo-model risk assessment and guideline concordance are presented in e-Tables 2 and 3, respectively.

The next diagnostic procedure ordered following clinician assessment, as well as the most invasive procedures used over the course of nodule management, are presented in Table 3. There was no difference in the immediate use of surgery between the intermediate- and high-risk pCA groups; however, those with a low pCA did not go on to surgery initially. Biopsy as the first diagnostic procedure was used similarly among all categories of clinician-assessed pCA. As the pretest probability for malignancy increased, so did the use of PET scans $(P<.001)$. As the pCA decreased, the use of CT surveillance as the initial management step increased 
TABLE 1] Demographics and Procedure Use Stratified by Diagnosis

\begin{tabular}{|c|c|c|c|c|}
\hline Variable & All Patients & Cancer & Benign & $P$ Value \\
\hline No. of patients & 337 & 158 & 179 & \\
\hline Age, y & $66.3( \pm 1.1)$ & $67.5( \pm 1.4)$ & $65.3( \pm 1.5)$ & .042 \\
\hline \multicolumn{5}{|l|}{ Sex, No. (\%) } \\
\hline Male & $176(52.2)$ & $74(46.8)$ & $102(57.0)$ & .063 \\
\hline Female & $161(47.8)$ & $84(53.2)$ & $77(43.0)$ & \\
\hline \multicolumn{5}{|l|}{ Smoking history status, No. (\%) } \\
\hline Never & $61(18.1)$ & $19(12.0)$ & $42(23.5)$ & .004 \\
\hline Former & $178(52.8)$ & $82(51.9)$ & $96(53.6)$ & \\
\hline Current & $98(29.1)$ & $57(36.1)$ & $41(22.9)$ & \\
\hline Pack-year, mean & $45.4( \pm 3.8)$ & $46.6( \pm 4.4)$ & $44.3( \pm 6.3)$ & .560 \\
\hline Lung nodule size (millimeters) & $15.8( \pm 0.6)$ & $18.4( \pm 0.9)$ & $13.6( \pm .7)$ & $<.001$ \\
\hline \multicolumn{5}{|l|}{$\begin{array}{l}\text { Next procedure after initial CT scan, } \\
\text { No. }(\%)\end{array}$} \\
\hline Surgery & $8(2.37)$ & $5(3.16)$ & $3(1.68)$ & .376 \\
\hline Biopsy & $30(8.90)$ & $14(8.86)$ & $16(8.94)$ & .981 \\
\hline CT scan & $96(28.49)$ & $12(7.59)$ & $84(46.93)$ & $<.001$ \\
\hline $\mathrm{PET} \leq 30 \mathrm{~d}$ before surgery & $43(12.76)$ & $37(23.42)$ & $6(3.35)$ & $<.001$ \\
\hline $\mathrm{PET}^{\mathrm{a}}$ & $160(47.48)$ & $90(56.96)$ & $70(39.11)$ & .018 \\
\hline \multicolumn{5}{|l|}{ Procedure use, No. (\%) } \\
\hline Surgery & $84(24.9)$ & $68(43.0)$ & $16(8.9)$ & $<.001$ \\
\hline Biopsy & $66(19.6)$ & $37(23.4)$ & $29(16.2)$ & .135 \\
\hline Biopsy and surgery & $58(17.2)$ & $50(31.6)$ & $8(4.5)$ & $<.001$ \\
\hline CT imaging only & $129(38.3)$ & $3(1.9)$ & $126(7.4)$ & $<.001$ \\
\hline \multicolumn{5}{|l|}{ PET use, No. (\%) } \\
\hline Overall PET & $227(67.4)$ & $142(89.9)$ & $85(47.5)$ & $<.001$ \\
\hline$\leq 30 \mathrm{~d}$ before surgery & $44(13.1)$ & $38(24.1)$ & $6(3.4)$ & $<.001$ \\
\hline$\leq 30 \mathrm{~d}$ after biopsy & $7(2.1)$ & $7(4.4)$ & $0(0.0)$ & .005 \\
\hline $\begin{array}{l}\leq 30 \mathrm{~d} \text { after biopsy and } \leq 30 \mathrm{~d} \\
\text { before surgery }\end{array}$ & $3(0.9)$ & $3(1.9)$ & $0(0.0)$ & .065 \\
\hline No relation to biopsy/surgery & $173(51.3)$ & $94(59.5)$ & $79(44.1)$ & .050 \\
\hline
\end{tabular}

aPET not performed within $30 \mathrm{~d}$ of biopsy or surgery

$(P<.001)$; however, $7 \%$ of subjects $(\mathrm{n}=10)$ in the highpCA category had CT imaging performed as the next step, contrary to guideline recommendations.

\section{Discussion}

The identification of incidentally detected pulmonary nodules is rising exponentially due to widespread use of CT scans. Although guidelines exist to aid in the management of pulmonary nodules, these continue to pose a diagnostic dilemma for clinicians. To our knowledge, this is the first study to prospectively follow the diagnostic odyssey of incidentally detected pulmonary nodules in the context of clinician assessed pretest pCA. It expands on a previously published analysis on nodule management by community pulmonologists and has several important findings. First, clinician-assessed pCA is better at predicting malignancy than two previously validated nodule calculators. Second, despite being more accurate at predicting malignancy, the majority of clinicians did not follow pCA-based guideline recommendations for nodule management when ordering the next test. Finally, clinicians were more aggressive than recommended for subjects in the low-risk category, in whom the vast majority of nodules were benign and serial surveillance was indicated. However, they were more conservative in their management decisions for patients in the high-risk category and thereby avoided recommending unnecessary surgery in a significant proportion of subjects with benign disease. 


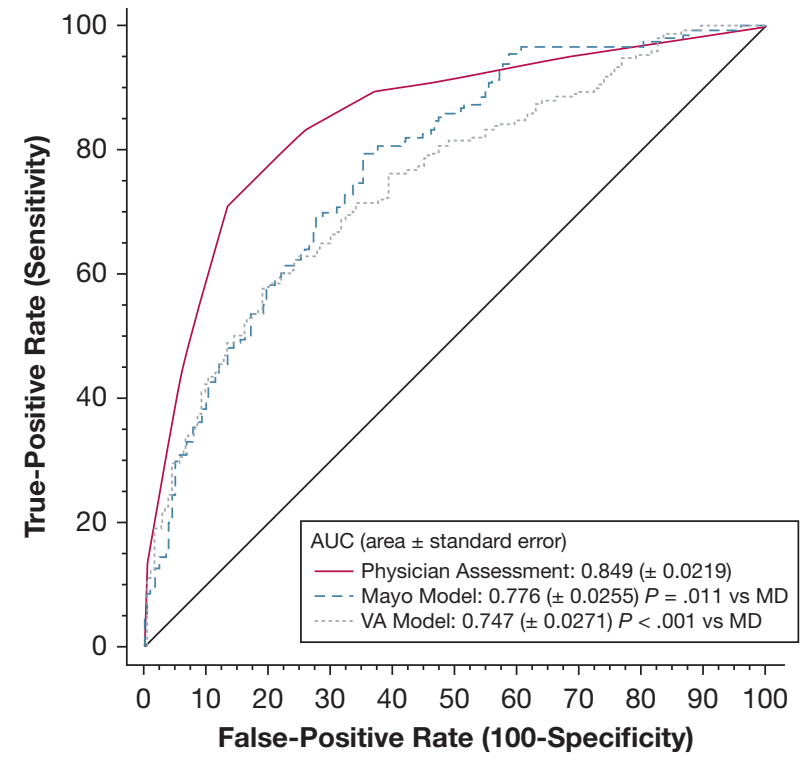

Figure 2 - Physician and model receiver operator characteristic curve accuracy.

Clinical practice guidelines have been available for more than a decade to assist clinicians in the management of pulmonary nodules. ${ }^{3,7,8}$ For patients with larger nodules ( $\geq 8 \mathrm{~mm}$ ), management decisions are based on an assessment of the pretest probability of cancer, which is determined by either the use of validated nodule prediction models or physician assessment. The two most widely used models include the Mayo Clinic model and the VA model. ${ }^{5,6}$ These incorporate clinical and radiographic factors, including size, border contour, location, patient age, smoking history, and prior history of cancer. They are partially dependent on the prevalence of cancer in the cohort in which they were originally developed. For example, the Brock model, developed from a screened population, had a prevalence of malignancy of $5.5 \%$ and performs well in smaller nodules (AUC, 0.91 in nodules $<10 \mathrm{~mm}$ ). ${ }^{9}$ The prevalence of malignancy in this cohort of patients was $47 \%$ and most closely mirrors the VA prediction model, in which prevalence was $54 \% .^{6}$ Overall, these models have been shown to be both accurate and useful in predicting the probability of cancer in pulmonary nodules. Although they have been compared with one another, ${ }^{10}$ to our knowledge, this is the first study that prospectively compares internally derived physician assessment to validated models and finds that physicians are highly accurate at predicting whether or not a nodule is malignant. These findings differ from a previous study in which physicians were presented with clinical vignettes and accompanying radiographs of patients with pulmonary nodules. ${ }^{11}$ That study showed that although clinicians and validated models had similar accuracy for characterizing pulmonary nodules, the accuracy was only fair to good for both. ${ }^{11}$ Another study compared predictions of the Mayo model with that of four expert clinicians (chest radiologist, pulmonologist, internist, and thoracic surgeon). Each clinician reviewed clinical information and plain chest radiographs from 100 selected cases and made a prediction of malignancy on a 10-point scale. In this study, there was no difference between the two; however, the accuracy was good for both. $^{12}$

In a previous study, we compared model-assessed pCA and guideline-directed management of pulmonary nodules in a geographically diverse sample of

TABLE 2 ] Physician Risk Assessment and Guideline Concordance

\begin{tabular}{|c|c|c|c|}
\hline Physician-Assessed Pretest Probability & All $n=25(\%)$ & Cancer $\mathrm{n}=3(\%)$ & Benign $\mathrm{n}=22(\%)$ \\
\hline \multicolumn{4}{|l|}{ Pretest probability $\leq 5 \%$} \\
\hline Guideline concordant CT surveillance & $12(48.0)$ & $0(0.0)$ & $12(54.5)$ \\
\hline More aggressive & $13(52.0)$ & $3(100.0)$ & $10(45.5)$ \\
\hline PET & $9(36.0)$ & $2(66.7)$ & $7(31.8)$ \\
\hline $\mathrm{PET} \leq 30 \mathrm{~d}$ before surgery & $1(4.0)$ & $1(33.3)$ & $0(0.0)$ \\
\hline \multirow[t]{2}{*}{ Biopsy } & $3(12.0)$ & $0(0.0)$ & $3(13.6)$ \\
\hline & All $n=138(\%)$ & Cancer $n=114(\%)$ & Benign $\mathrm{n}=24(\%)$ \\
\hline \multicolumn{4}{|l|}{ Pretest probability $\geq 60 \%$} \\
\hline Guideline-concordant surgery ${ }^{a}$ & $35(25.4)$ & $33(28.9)$ & $2(8.3)$ \\
\hline More conservative & $103(74.6)$ & $81(71.1)$ & $22(91.7)$ \\
\hline CT & $10(7.2)$ & $5(4.4)$ & $5(20.8)$ \\
\hline $\mathrm{PET}^{\mathrm{a}}$ & $78(56.5)$ & $63(55.3)$ & $15(62.5)$ \\
\hline Biopsy & $15(10.9)$ & $13(11.4)$ & $2(8.3)$ \\
\hline
\end{tabular}

aPET performed within $30 \mathrm{~d}$ of surgery was considered a staging PET scan and concordant with guidelines. 
TABLE 3 ] Physician Pretest Probability Assessment and Procedure Use Across Tertiles of Risk

\begin{tabular}{|c|c|c|c|c|}
\hline Demographics and Characteristics & $<0.05(n=25)$ & 0.05 to $<0.60(n=174)$ & $\geq 0.60(n=138)$ & $P$ Value \\
\hline Physician risk & $2.5(\mathrm{~N} / \mathrm{A})$ & $26.0( \pm 2.4)$ & $78.9( \pm 1.9)$ & \\
\hline VA risk for same group & $34.1( \pm 8.8)$ & $36.5( \pm 3.2)$ & $59.8( \pm 3.4)$ & \\
\hline Mayo risk for same group & $24.8( \pm 8.6)$ & $29.8( \pm 3.1)$ & $53.1( \pm 4.1)$ & \\
\hline \multicolumn{5}{|l|}{ Outcome, No. (\%) } \\
\hline Benign & $22(88.0)$ & $133(76.4)$ & $24(17.4)$ & $<.001$ \\
\hline Cancer & $3(12.0)$ & $41(23.6)$ & $114(82.6)$ & \\
\hline Age, y & $67.5( \pm 4.7)$ & $65.3( \pm 1.5)$ & $67.4( \pm 1.5)$ & .144 \\
\hline \multicolumn{5}{|l|}{ Sex, No. (\%) } \\
\hline Male & $14(56.0)$ & $92(52.9)$ & $70(5.7)$ & .862 \\
\hline Female & $11(44.0)$ & $82(47.1)$ & $68(49.3)$ & \\
\hline Smoking history status, No. (\%) & & & & .003 \\
\hline Never & $7(28.0)$ & $41(23.6)$ & $13(9.4)$ & \\
\hline Former & $13(52.0)$ & $92(52.9)$ & $73(52.9)$ & \\
\hline Current & $5(20.0)$ & $41(23.6)$ & $52(37.7)$ & \\
\hline Pack-year, mean & $40.6( \pm 15.9)$ & $41.6( \pm 5.0)$ & $49.5( \pm 5.0)$ & .106 \\
\hline Abstinence, y & $10.6( \pm 6.3)$ & $9.6( \pm 2.2)$ & $5.7( \pm 1.7)$ & .020 \\
\hline Lung nodule size & $12.2( \pm 1.2)$ & $14.2( \pm 0.8)$ & $18.5( \pm 1.0)$ & $<.001$ \\
\hline \multicolumn{5}{|l|}{$\begin{array}{l}\text { Next procedure after initial CT } \\
\text { scan, No. }(\%)\end{array}$} \\
\hline Surgery & $0(0.0)$ & $3(1.7)$ & $5(3.6)$ & .409 \\
\hline Biopsy & $3(12.0)$ & $12(6.9)$ & $15(1.9)$ & .453 \\
\hline CT scan & $12(48.0)$ & $74(42.5)$ & $10(7.2)$ & $<.001$ \\
\hline $\mathrm{PET} \leq 30 \mathrm{~d}$ before surgery & $1(4.0 \%)$ & $12(6.9)$ & $30(21.7)$ & $<.001$ \\
\hline $\mathrm{PET}^{\mathrm{a}}$ & $9(36.0)$ & $73(42.0)$ & $78(56.5)$ & $<.001$ \\
\hline \multicolumn{5}{|l|}{ Total procedure use, No. (\%) } \\
\hline Surgery only & $2(8.0)$ & $29(16.7)$ & $52(37.7)$ & $<.001$ \\
\hline Biopsy only & $3(12.0)$ & $32(18.4)$ & $31(22.5)$ & .496 \\
\hline Biopsy and surgery & $2(8.0)$ & $12(6.9)$ & $44(31.9)$ & $<.001$ \\
\hline Biopsy or surgery & $5(20.0)$ & $61(35.1)$ & $83(60.1)$ & $<.001$ \\
\hline PET & $10(40.0)$ & $95(54.6)$ & $122(88.4)$ & $<.001$ \\
\hline CT scan only & $18(72.0)$ & $99(56.9)$ & $11(8.0)$ & $<.001$ \\
\hline \multicolumn{5}{|l|}{ Procedure use category, No. (\%) } \\
\hline Most invasive $=$ surgery & $4(16.0)$ & $41(23.6)$ & $96(69.6)$ & $<.001$ \\
\hline Most Invasive $=$ biopsy & $3(12.0)$ & $32(18.4)$ & $31(22.5)$ & .496 \\
\hline Surveillance & $19(72.0)$ & 99 (56.9) & $11(8.0)$ & $<.001$ \\
\hline
\end{tabular}

aPET not performed within $30 \mathrm{~d}$ of biopsy or surgery

community pulmonologists. ${ }^{4}$ That study demonstrated that despite recommendations for management based on risk, physicians did not follow guidelines. For example, $44 \%$ of patients with low pCA underwent invasive testing when surveillance with serial CT imaging was indicated. Further, there was no difference in the frequency of surgery among patients with a low, intermediate, or high risk of cancer, and surgery was performed in $35 \%$ of patients with benign disease. ${ }^{4}$ This finding was not seen in the current study, in which the use of surgery appropriately increased as the probability of cancer increased, and surveillance proportionally decreased. An important limitation to the previous study was the fact that there was no way of discerning if clinicians used their clinical intuition, a model, both, or neither to direct nodule management decisions. The findings of both of these studies are consistent with the literature published from the VA hospital system, in 
which half of patients with incidentally detected nodules and a pCA $<65 \%$ received guidelineconcordant care. ${ }^{13}$ It may be that clinicians do not find the guidelines valuable, given that many of the recommendations for nodule management are grade $2 \mathrm{C}$ (weak recommendation, low-quality evidence). ${ }^{3,14}$

In the current study, clinician assessment of pCA was collected prospectively and prior to nodule management decisions. Despite accurate clinical intuition, physicians did not follow guideline-based recommendations for nodule management when selecting the next diagnostic test. They "erred" in both directions. In the low-risk category, they were more aggressive when serial imaging would suffice. In this category of risk, all but three patients had benign disease, yet 52\% were managed more aggressively than recommended with either PET or biopsy. Thus, using physician intuition and following the guidelines would have provided more optimal care.

Conversely, in patients in whom the pCA exceeded $60 \%$ and guidelines recommend surgery, clinicians opted for more conservative management with biopsy, CT imaging, or PET scanning 75\% of the time. In doing so, surgery was avoided in 22 of 24 patients with benign nodules. However, there was a trade-off in that $11 \%$ of those with cancer $(\mathrm{n}=13)$ underwent an unnecessary biopsy procedure prior to surgery. The British Thoracic Society guidelines differ from the third iteration of the ACCP guidelines for nodule management in that the threshold for high risk is set at $>70 \%$ vs $\geq 65 \%$. ${ }^{2,3}$ One study using Markov modeling to determine appropriate thresholds for deciding management strategies for patients unfit for surgery suggested that $\mathrm{pCA} \geq 85 \%$ is appropriate to proceed to treatment without biopsyproven cancer. ${ }^{15}$ Our findings support the higher threshold for categorizing a nodule as high risk and suggest that further adjustment to the current guidelines may be indicated. For example, shifting the cutoff of pCA that categorizes a nodule as high risk (direct to surgery with no further testing) to $80 \%$ or greater would decrease the number of operations performed for patients with benign nodules if clinicians were to strictly follow the guidelines. This would come at the expense of a few additional biopsies in those with cancer. Models using this and other data could optimize nodule management by better defining low-, intermediate-, and high-risk categories and should be an area of future research.
This study has a number of limitations. First, we were unable to ascertain the degree to which patient preference may have played a role in management decisions regarding the nodule. Future studies should capture patient preferences and willingness to follow guidelines for nodule management. Second, the overall study was designed to test a blood biomarker, and thus the physicians at those study sites may have had more expertise in nodule management, perhaps limiting the generalizability of these findings. In addition, patient comorbidities were not accounted for in the rationale behind management decisions; it is possible that those with a high-risk pretest probability for malignancy were not surgical candidates and therefore underwent a biopsy procedure prior to radiation treatment or were managed more conservatively with imaging. Finally, there are additional nodule prediction models that incorporate PET information (Herder) and volumetric measurements (Bayesian Inference Malignancy Calculator) that may be more accurate than the prediction models used here; however, we did not have PET data on all patients, and volumetric measurements were not provided. ${ }^{16-20}$ The strengths of the study include the fact that it is a geographically diverse sample with prospective data collected from patients with pulmonary nodules. In addition, an important attribute of this study was that physician assessment of pCA prior to diagnostic testing was provided.

In conclusion, clinicians are adept at distinguishing benign from malignant pulmonary nodules; however, they do not apply that data to the guidelines for nodule management consistently. In some instances, straying from the guidelines subjected patients to unnecessary testing, whereas in others, it appeared to prevent surgery for benign nodules. These findings have implications for patients presenting with pulmonary nodules, an increasingly common problem facing clinicians and patients. Enhancing existing prediction calculators by including novel radiographic measurements and exhaled, serum, and bronchoscopic biomarkers may aid in distinguishing benign from malignant disease and is an area of ongoing research. ${ }^{21-24}$ Until these technologies become available or more widely used, more focus is needed in the areas of guideline refinement, implementation, and dissemination to provide optimal patient care. 


\section{Acknowledgments}

Author contributions: N. T. T., A. P., and G. A. S. contributed to the design of the paper, analysis and interpretation of the data, drafting the paper, and final approval. They agree to be accountable for all aspects of the work. X. L. contributed to the analysis and interpretation of the data, drafting the paper, final approval, and accountability for all aspects of the work. M. K. G. and A. V. contributed to interpretation of data, drafting the paper, and final approval and agree to be accountable for all aspects of the work. G. A. S. and N. T. T. are guarantors of the paper.

Financial/nonfinancial disclosures: The authors have reported to CHEST the following: N. T. T. has received foundation grant funding from the American Cancer Society and the Chest Foundation; industry grant funding from Exact Sciences, Veracyte, Integrated Diagnostics, and Olympus; consulting monies from Integrated Diagnostics, Olympus, and Oncocyte; and has participated in advisory board meetings for Veracyte. A. P. and X-J. L. are employees of Integrated Diagnostics. M. G. has received a research award from PCORI, a research grant from NIH/NCI, and research support from Medial, Inc. A. V has served on advisory boards for Integrated Diagnostics, Veracyte, Oncocyte, and Nucleix. G. A. S. has received grant funding from Integrated Diagnostics, Exact Sciences, and Veracyte.

Role of the sponsors: The sponsor had no role in the design or direct collection of data for the study. The sponsor did participate in the analysis of data and preparation of the methods and tables for the manuscript.

Additional information: The e-Appendix, e-Figure, and e-Tables can be found in the Supplemental Materials section of the online article.

\section{References}

1. Gould MK, Tang T, Liu IL, et al. Recent trends in the identification of incidental pulmonary nodules. Am J Respir Crit Care Med. 2015;192(10):1208-1214.

2. Baldwin DR, Callister ME; Guideline Development Group. The British Thoracic Society guidelines on the investigation and management of pulmonary nodules. Thorax. 2015;70(8):794-798.

3. Gould MK, Donington J, Lynch WR, et al. Evaluation of individuals with pulmonary nodules: when is it lung cancer? Diagnosis and management of lung cancer, 3rd ed: American College of Chest Physicians evidence-based clinical practice guidelines. Chest. 2013;143(5 suppl):e93S-e120S.
4. Tanner NT, Aggarwal J, Gould MK, et al. Management of pulmonary nodules by community pulmonologists: a multicenter observational study. Chest. 2015;148(6): 1405-1414.

5. Swensen SJ, Silverstein MD, Ilstrup DM, Schleck CD, Edell ES. The probability of malignancy in solitary pulmonary nodules. Application to small radiologically indeterminate nodules. Arch Intern Med. 1997;157(8):849-855.

6. Gould MK, Ananth L, Barnett PG; Veterans Affairs SNAP Cooperative Study Group. A clinical model to estimate the pretest probability of lung cancer in patients with solitary pulmonary nodules. Chest. 2007;131(2):383-388.

7. Gould MK, Fletcher J, Iannettoni MD, et al. Evaluation of patients with pulmonary nodules: when is it lung cancer?: ACCP evidence-based clinical practice guidelines (2nd edition). Chest. 2007;132(3 suppl):108S-130S.

8. Callister ME, Baldwin DR, Akram AR, et al. British Thoracic Society guidelines for the investigation and management of pulmonary nodules. Thorax. 2015;70(suppl 2):ii1-ii54.

9. McWilliams A, Tammemagi MC, Mayo JR, et al. Probability of cancer in pulmonary nodules detected on first screening CT. N Engl J Med. 2013;369(10): 910-919.

10. Schultz EM, Sanders GD, Trotter PR, et al. Validation of two models to estimate the probability of malignancy in patients with solitary pulmonary nodules. Thorax. 2008;63(4):335-341.

11. Balekian AA, Silvestri GA, Simkovich SM, et al. Accuracy of clinicians and models for estimating the probability that a pulmonary nodule is malignant. Ann Am Thorac Soc. 2013;10(6):629-635.

12. Swensen SJ, Silverstein MD, Edell ES, et al. Solitary pulmonary nodules: clinical prediction model versus physicians. Mayo Clin Proc. 1999;74(4):319-329.

13. Wiener RS, Gould MK, Slatore CG, Fincke BG, Schwartz LM, Woloshin S. Resource use and guideline concordance in evaluation of pulmonary nodules for cancer: too much and too little care. JAMA Intern Med. 2014;174(6): 871-880.

14. Guyatt G, Gutterman D, Baumann MH, et al. Grading strength of recommendations and quality of evidence in clinical guidelines: report from an American College of Chest Physicians task force. Chest. 2006;129(1):174-181.
15. Louie AV, Senan S, Patel P, et al. When is a biopsy-proven diagnosis necessary before stereotactic ablative radiotherapy for lung cancer? A decision analysis. Chest. 2014;146(4):1021-1028.

16. Perandini S, Soardi GA, Motton M, Rossi A, Signorini M, Montemezzi S. Solid pulmonary nodule risk assessment and decision analysis: comparison of four prediction models in 285 cases. Eur Radiol. 2016;26(9): 3071-3076.

17. Soardi GA, Perandini S, Motton M, Montemezzi S. Assessing probability of malignancy in solid solitary pulmonary nodules with a new Bayesian calculator: improving diagnostic accuracy by means of expanded and updated features. Eur Radiol. 2015;25(1):155-162.

18. Al-Ameri A, Malhotra $\mathrm{P}$, Thygesen $\mathrm{H}$, et al. Risk of malignancy in pulmonary nodules: a validation study of four prediction models. Lung Cancer. 2015;89(1):27-30.

19. Perandini S, Soardi GA, Larici AR, et al. Multicenter external validation of two malignancy risk prediction models in patients undergoing 18F-FDG-PET for solitary pulmonary nodule evaluation [published online ahead of print September 15, 2016]. Eur Radiol. E-pub ahead of print.

20. Herder GJ, van Tinteren H, Golding RP, et al. Clinical prediction model to characterize pulmonary nodules: validation and added value of $18 \mathrm{~F}$ fluorodeoxyglucose positron emission tomography. Chest. 2005;128(4): 2490-2496.

21. Silvestri GA, Vachani A, Whitney D, et al. A bronchial genomic classifier for the diagnostic evaluation of lung cancer. N Engl J Med. 2015;373(3):243-251.

22. Vachani A, Pass HI, Rom WN, et al. Validation of a multiprotein plasma classifier to identify benign lung nodules. I Thorac Oncol. 2015;10(4):629-637.

23. Mazzone PJ, Wang XF, Xu Y, et al. Exhaled breath analysis with a colorimetric sensor array for the identification and characterization of lung cancer. J Thorac Oncol. 2012;7(1):137-142.

24. Massion PP, Healey GF, Peek LJ, et al. Autoantibody signature enhances the positive predictive power of computed tomography and nodule-based risk models for detection of lung cancer [published online ahead of print September 8, 2016]. J Thorac Oncol. http://dx.doi.org/10.1016/j.jtho.2016. 08.143 